\title{
Management of non-maturing deposits by multistage stochastic programming
}

\author{
Karl Frauendorfer* Michael Schürle ${ }^{\dagger}$ \\ University of St. Gallen \\ Institute of Operations Research \\ Holzstrasse 15 \\ CH-9010 St. Gallen \\ Switzerland
}

August 7, 2002

\begin{abstract}
The management of non-maturing account positions in a bank's balance like savings and sight deposits as well as certain types of variable-rate mortgages is complicated by the embedded options that its clients may exercise. In addition to the usual interest rate risk, uncertainty in the timing and amount of cash flows must be taken into account when investment or refinancing strategies are determined. This paper introduces a multistage stochastic programming model where
\end{abstract}

*email: karl.frauendorfer@unisg.ch, phone: +41 712242101 (corresponding author)

$\dagger$ email: michael.schuerle@unisg.ch 
the stochastic evolution of interest rates and volume under management is described by stochastic processes in discrete time. Scenarios are generated by means of barycentric approximation which is particularly useful to deal with the observed correlations between interest rates and volume. Practical experience from the application at a major Swiss bank is reported where the model has been employed since the mid-90s.

Keywords: Stochastic programming; Banking; Asset and liability management

\section{Introduction}

A significant portion of assets and liabilities within a bank's balance typically consists of so-called "non-maturing accounts". This includes savings and sight deposits on the liability side as well as certain types of variable-rate mortgages which are common in some countries on the asset side. A characteristic feature of these positions is that they have no contractual maturity. Instead, bank clients may freely increase or withdraw their investments or repay their mortgages, respectively, at any point in time at no penalty. On the other hand, the bank is allowed to adjust the rate that investors receive or borrowers must pay as a matter of policy. As a consequence, the volume of a non-maturing account may fluctuate heavily as clients react to changes in the market environment like rising or falling interest rates, the relative attractiveness of alternative investment or financing opportunities etc.

For example, during a period of high market rates, bank clients shift their investments in variable-rate deposits to fix-rate bonds with long maturities. In case of low interest rates, the volume increases since yields on savings accounts become relatively attractive compared to short-term securities, and 
even some institutional investors like pension funds prefer such deposits to direct investments in money market instruments. As a consequence, a negative correlation between interest rates and the total volume of the position can be observed. Moreover, it may become difficult to find a portfolio of fixed-income instruments that provides a sufficient margin and takes the risk into account that a significant portion of the savings volume is withdrawn (liquidity risk).

Analogously, there is a positive correlation between interest rates and the volume of variable-rate mortgages in a period of high yields when refinancing on the market is unfavorable for the bank. In contrast to this, clients switch to fixed maturities when interest rates are low in order to hedge themselves against a future rise (prepayment risk). These difficulties caused a broad discussion among practitioners from the financial industry about the management of non-maturing accounts and the risks induced by the embedded options.

This article proposes a multistage stochastic programming model for the determination of optimal investment and refinancing strategies. In the formulation of the stochastic program, uncertainty in future interest rates affects the coefficients in the objective while the stochastic volume change enters the right-hand-side of the constraints. Representative scenarios for these risk factors are generated by means of the barycentric approximation scheme that is particularly useful to address the observed correlations between interest rates and volume. The existence of certain structural properties which are discussed in the sequel allows to obtain exact bounds for the underlying problem given by the solution of convex multistage stochastic programs. Since the scenarios are based on current market observations, the resulting policies are adjusted dynamically over time to the present situation. In this way, 
the stochastic optimization model helps overcome many limitations of static approaches as they are used in the financial industry today.

The remainder of this paper is organized as follows: In the next section, shortcomings of traditional techniques caused by the uncertain maturities are discussed which do not apply to the dynamic approach. The optimization model for the management of non-maturing accounts is formulated in Section 3. After a discussion of relevant properties of the underlying stochastic program, Section 4 introduces the barycentric approximation scheme that is used here for scenario generation. Results of a case study are reported in Section 5. The final Section 6 summarizes practical experience from the application at a major Swiss bank and gives an outlook to possible improvements of the model and future directions of research.

\section{The problem of uncertain maturities}

\subsection{Traditional approaches}

It is often argued that a bank's balance sheet may be viewed as a long position (assets) and a short position (liablities) in bonds of different maturities. Duration matching is a widely used technique within asset and liability management (ALM). In the classical sense, it hedges against small parallel shifts of the yield curve by matching the interest rate sensitivity of the positions on both sides of the balance, larger changes can be immunized by taking convexity into account (see [24] for example). In order to reflect more complex interest rate movements, various extensions of this concept have been introduced, e.g., the key rate durations due to Ho [16]. However, duration based approaches require a permanent rebalancing of the portfolio, particularly in the presence of implicit options, which induces significant transaction 
costs. In case of non-maturing accounts, it is also difficult to find adequate duration measures. The risks induced by the stochastic nature of cash flows cannot be aggregated into a single number (duration), at least not without additional (and often inadequate) assumptions on the timing of payments. Similar problems arise in gap analysis [24] where positions are assigned to time bands.

These shortcomings have motivated the replicating portfolio approach [18]. Instead of identifying and hedging against the various sources of risk, one tries to mimic the behavior of the target account in order to capture its characteristics. This is achieved by determination of a portfolio of fixed income securities whose return replicates the client rate of the relevant asset or liability position plus a margin. Transaction costs remain low since liquid money market instruments and swaps are used that are held until maturity to avoid rebalancing activities.

In the application of this strategy, maturing funds are always renewed at the same maturity. Whenever the volume of the position under management increases or decreases due to changes in client demand, funds are bought or sold at constant proportions. These weights are derived by minimizing the tracking error, i.e., the difference between the average portfolio return and the client rate, over a historic sample period under the constraint that the volume of the replicating portfolio matches that of the target account at all points in time. In this way, liquidity and prepayment risks are implicitly taken into account.

By means of the approaches mentioned so far, uncertain cash flows are transformed into (apparently) certain ones, allowing the bank to manage them like normal positions with deterministic maturities. However, the considerable risk of an incorrect transformation remains. For example, different 
historic sample periods for the determination of a replicating portfolio may result in substantially different portfolio weights. Moreover, these techniques are static in the sense that they do not incorporate the possibility of future changes in random data (here: interest rates, volume) and their impact on the optimal portfolio composition. Therefore, the question arises if a $d y$ namic policy with active reactions to changes in the market environment and client behavior could increase the bank's profit. In particular, it remains to be clarified if the observed correlations between interest rates and volume can be exploited more appropriately to manage the different risks associated with non-maturing account positions.

\subsection{Stochastic programming models}

Stochastic programming models overcome the limitations of static approaches described above. First, they incorporate the uncertainty in interest rates and volume by generating scenarios of their possible future outcomes. These scenarios quantify the impact of changes in the underlying risk factors on the return of investment or refinancing strategies. Second, a multistage stochastic program reflects not only the dynamics of uncertain data but also of decisions more appropriately since transactions may take place at discrete points in time over some finite planning horizon. For all scenarios under consideration, a decision must be taken at each stage based on realizations of random data and earlier decisions. This allows the correction of an initial policy, e.g., if it fails to achieve the investment goal for certain scenarios. Third, various kinds of constraints can easily be taken into account, e.g., to ensure a desired structure of the portfolio or to reduce the risk exposure with respect to certain positions etc.

Meanwhile, a large number of stochastic optimization models has been 
developed for various applications in Finance. Among them are the RussellYasuda-Kasai model for asset allocation due to Cariño and Ziemba [3], which can be seen as the first successful commercial application of multistage stochastic programming, the models for ALM and (fixed-income) portfolio management of Mulvey $[20,21]$ and Zenios et al. $[1,15]$, or the multistage portfolio optimization model due to Dantzig and Infanger [4], to mention just a few.

The model introduced in the sequel has been applied for several years by a major Swiss bank for the management of its deposits and variable-rate mortgages. For simplicity, only the problem of reinvesting savings positions in the money and capital markets is investigated. The model for refinancing mortgages is equivalent and can be easily derived from the formulation discussed here.

\section{The stochastic optimization model}

\subsection{Formulation as linear program}

The formulation of the optimization model is straightforward: Let $\mathcal{D}=$ $\{1,2, \ldots, D\}$ denote a set of points in time where fixed-income securities held in the portfolio mature ( $D$ is the longest available maturity). Investment opportunities are given by a set of traded standard maturities $\mathcal{D}^{S} \subseteq \mathcal{D}$. Unless not restricted otherwise, the model has also the option to raise funds which are reinvested in addition to the actual volume of the non-maturing account. For the investment of $\$ 1$ in maturity $d \in \mathcal{D}^{S}$ at time $t$, one receives accrued discounted interest payments of $\varphi_{t}^{d,+}$ while costs of $\varphi_{t}^{d,-}$ arise for short positions.

Obviously, for $t>0$ these coefficients depend on future interest rates and, hence, are uncertain. It is assumed that $\varphi_{t}^{d,+}\left(\eta_{t}\right)$ and $\varphi_{t}^{d,-}\left(\eta_{t}\right)$ for all $d \in \mathcal{D}^{S}$ 
are functions of a $K_{t}$-dimensional stochastic process $\left(\eta_{t} ; t=1, \ldots, T\right)$ in discrete time driving the evolution of the term structure. Here, $K_{t}=K=3$ correlated Brownian motions with (possibly time-dependent) drift are used that correspond to three key rates of different maturities, allowing the model to reflect shift, tilt, and humped movements of the term structure. Rates for the remaining (non-key rate) maturities are interpolated. Intuitively, this is based on the idea that the yield curve can be segmented into different sections where interest rates move in the same direction (see Ho [16]).

The sensitivity of interest rates subject to changes in the risk factors, transactions costs (bid/ask spread), as well as a discount mechanism are incorporated by the functional relationship between $\eta_{t}$ and $\varphi_{t}^{d,+}, \varphi_{t}^{d,-}$. A formal specification of the relation between risk factors and coefficients is omitted here since its notation becomes rather cumbersome. The stochastic volume change is also modelled by a Brownian motion in discrete time $\left(\xi_{t} ; t=\right.$ $1, \ldots, T)$ of dimension $L_{t}=L=1$. It may be correlated with the components of the stochastic process $\eta_{t}$ to reflect the dependency between changes in interest rates and volume.

At each point in time $t=0, \ldots, T$, decisions on the amount $x_{t}^{d,+} \geq 0$ of long and $x_{t}^{d,-} \geq 0$ of short sales in maturity $d \in \mathcal{D}^{S}$ have to be made. The objective is to maximize the expected present value of the income from all investments $\varphi_{t}^{d,+} \cdot x_{t}^{d,+}$ minus the costs for borrowings $\varphi_{t}^{d,-} \cdot x_{t}^{d,-}$ for all $d \in \mathcal{D}^{S}$ over the planning horizon $T$. If the expectation is taken with respect to the joint probability measure $P$ of the risk factors $(\eta, \xi)$, where $\eta:=\left(\eta_{1}, \ldots, \eta_{T}\right)$, $\xi:=\left(\xi_{1}, \ldots, \xi_{T}\right)$, and $\Theta \times \Xi$ is the corresponding support, the optimization problem reads as: 


$$
\begin{aligned}
& \max \int_{\Theta \times \Xi} \sum_{t=0}^{T} \sum_{d \in \mathcal{D}^{S}}\left(\varphi_{t}^{d,+}\left(\eta_{t}\right) \cdot x_{t}^{d,+}-\varphi_{t}^{d,-}\left(\eta_{t}\right) \cdot x_{t}^{d,-}\right) d P(\eta, \xi) \\
& \text { s.t. (1a) } x_{t}^{d}-x_{t-1}^{d+1} \quad=0 \quad t=0, \ldots, T ; \forall d \notin \mathcal{D}^{S} \\
& \text { (1b) } \quad x_{t}^{d}-x_{t-1}^{d+1}-x_{t}^{d,+}+x_{t}^{d,-}=0 \quad t=0, \ldots, T ; \forall d \in \mathcal{D}^{S} \\
& \text { (2) } v_{t}-\sum_{d \in \mathcal{D}} x_{t}^{d} \quad=0 \quad t=0, \ldots, T \\
& \text { (3) } v_{t}-v_{t-1} \quad=\xi_{t} \quad t=1, \ldots, T \\
& \text { (4) } \sum_{d \in \mathcal{D}^{S}} x_{t}^{d,+}-\sum_{d=1}^{m_{t}} x_{t-1}^{d} \leq \xi_{t} \quad t=0, \ldots, T \\
& \text { (5a) } 0 \leq x_{t}^{d,+} \leq \ell_{t}^{d,+} \quad t=0, \ldots, T ; \forall d \in \mathcal{D}^{S} \\
& \text { (5b) } \quad 0 \leq x_{t}^{d,-} \leq \ell_{t}^{d,-} \quad t=0, \ldots, T ; \forall d \in \mathcal{D}^{S} \\
& \text { (6a) } \quad x_{t}^{d,+}, x_{t}^{d,-} \quad \text { nonanticipative } \quad t=0, \ldots, T ; \forall d \in \mathcal{D}^{S} \\
& \text { (6b) } v_{t}, x_{t}^{d} \in \mathbb{R} \text { nonanticipative } \quad t=0, \ldots, T ; \forall d \in \mathcal{D} \text {. }
\end{aligned}
$$

Budget constraint (1a) ensures that the (net) sum $x_{t}^{d} \in \mathbb{R}$ of all investments minus borrowings maturing after $d$ periods equals the corresponding value in the previous period for non-traded maturity dates while (1b) corrects it by the new long and short sales in $t$ for traded maturities. Herein, $x_{-1}^{d}$ denotes the amount initially held in maturity $d$ from decisions in the past. Constraint (2) requires that the portfolio matches the total volume $v_{t}$ of the non-maturing account position while the latter is given by the stochastic volume change according to (3).

Raising short positions and reinvesting them in addition to the volume of the non-maturing account may be viewed as some speculative strategy that is beyond the actual scope of the application. Therefore, (4) restricts the investment in $t$ to the amount of funds maturing within the next $m_{t}>0$ 
periods. Note that this controls short sales indirectly through the budget constraints. While $m_{t}=1$ prohibits any short sales in $t$, raising liabilities might be required to achieve feasibility of the problem when a large decrease in the total volume occurs that cannot be compensated by the funds maturing at a certain stage. Liquidity restrictions which must be observed for some maturities $d \in \mathcal{D}^{S}$ particularly on the Swiss market are taken into account by upper limits $\ell_{t}^{d,+}$ for investments and $\ell_{t}^{d,-}$ for borrowings in (5a) and (5b).

The entire set of constraints must hold for all realizations of $\eta_{t}$ and $\xi_{t}$, $t=1, \ldots, T$. Moreover, investment policies at any stage $t>0$ have to be made independent of future outcomes of interest rates and volume, i.e., must not anticipate any information that becomes available in the future. These requirements are expressed by the so-called nonanticipativity constraints (6a) and (6b). Formally, their meaning is that decisions $x_{t}^{d,+}$ and $x_{t}^{d,-}$ depend only on the history of random data $\eta_{1}, \ldots, \eta_{t}$ and $\xi_{1}, \ldots, \xi_{t}$ but not on future realizations $\eta_{t+1}, \ldots, \eta_{T}$ or $\xi_{t+1}, \ldots, \xi_{T}$ since these are unknown at time $t$. An intuitive explanation is given in the next section.

\subsection{Scenario generation}

To evaluate the objective in (1) for a particular policy, it is necessary to calculate an integral with respect to the probability measure $P$. At each stage $t$, the profits and costs induced by a decision can be quantified by a value function that is formally introduced in Section 4.2. This function is not given analytically, even not explicitly, but implicitly by the solution of a multistage stochastic program with respect to the remaining stages $t+$ $1, \ldots, T$. Therefore, the integration in (1) cannot be performed analytically, and numerical methods are required.

[Figure 1 about here.] 
A common approach is the approximation of the original (continuous) distributions of random data at each stage of the stochastic program by discrete ones. This results in the generation of a scenario tree (see Figure 1 (a)) that is formally defined as

$$
\mathcal{A}:=\left\{(\eta, \xi) \in \Theta \times \Xi \mid\left(\eta_{t}, \xi_{t}\right) \in \mathcal{A}_{t}\left(\eta^{t-1}, \xi^{t-1}\right) \forall t>1\right\}
$$

In each node along the paths from the root to the leaves of the tree, one determines a set $\mathcal{A}_{t}\left(\eta^{t-1}, \xi^{t-1}\right)$ of finitely many outcomes for $\left(\eta_{t}, \xi_{t}\right)$ conditioned on the history of realizations $\eta^{t-1}:=\left(\eta_{1}, \ldots, \eta_{t-1}\right), \xi^{t-1}:=\left(\xi_{1}, \ldots, \xi^{t-1}\right)$. Such a scenario tree $\mathcal{A}$ is seen as "representative" for the entire universe of possible future outcomes of random data in the course of time. The corresponding conditional probabilities are denoted by $q_{t}\left(\cdot \mid \eta^{t-1}, \xi^{t-1}\right)$. Hence, the associated path probabilities are given by

$$
q(\eta, \xi):=\prod_{t=1}^{T} q_{t}\left(\eta_{t}, \xi_{t} \mid \eta^{t-1}, \xi^{t-1}\right) .
$$

As a result of the discretization, the integration in the objective of (1) is replaced by the calculation of a sum that can easily be performed. In this context, nonanticipativity constraints link those decision and state variables that share a common scenario up to a certain node (see Figure 1 (b)). Note that a scenario tree grows exponentially with respect to the number of stages, i.e., the planning horizon $T$ (curse of dimensionality), which requires a careful selection of scenarios to keep the problem size manageable.

To this end, certain properties of the stochastic program can be exploited that help control the scenario generation process and allow the determination of exact upper and lower bounds for the original problem. It can easily be seen from the formulation of the stochastic program that the interest rate risk factors $\eta_{t}, t=1, \ldots, T$, appear only in the objective while the volume change 
$\xi_{t}$ affects solely the right-hand side of constraints in (1). In particular, the coefficients on the left side are deterministic. Together with some additional assumptions which are discussed in the sequel, it is ensured that the value functions at $t$ are convex-concave saddle functions in $\left(\eta_{t}, \xi_{t}\right)$.

This saddle property motivates the application of barycentric approximation $[8,9,10]$. It starts from partitioning the domain of random data into simplicial cells for all stages. Bilinear upper and lower approximations to the corresponding value functions are constructed at distinguished points within these simplices, so-called generalized barycenters. An advantageous feature is that the discretization error can be quantified exactly for all nodes in the tree, and the accuracy may be improved by deliberately adding new scenarios (see [12]).

While a great variety of approximation methodologies exists (e.g., [2, 5, $6,11])$ that differ in the selection of support points and calculation of discrete probability measures, barycentric approximation is particularly useful for correlated distributions. The computational effort and the number of discrete outcomes at each stage is linear in the dimension of the underlying distribution. Therefore, the problem size remains moderate even for multistage programs when only a three-dimensional stochastic process is used for the description of the interest rate dynamics and a one-dimensional process for the volume as in the given case.

\section{Barycentric approximation}

\subsection{Formal description}

As outlined above, it is distinguished between those stochastic coefficients

$\eta_{t} \in \Theta_{t} \subset \mathbb{R}^{K_{t}}$ that influence the objective function and random data $\xi_{t} \in$ 
$\Xi_{t} \subset \mathbb{R}^{L_{t}}$ that appear only on the right-hand side of constraints for $t=$ $1, \ldots, T$. The support of $\eta=\left(\eta_{1}, \ldots, \eta_{T}\right)$ is denoted by $\Theta=\Theta_{1} \times \ldots \times \Theta_{T} \subset$ $\mathbb{R}^{K}, K=K_{1}+\ldots+K_{T}$, and that of $\xi=\left(\xi_{1}, \ldots, \xi_{T}\right)$ by $\Xi=\Xi_{1} \times \ldots \times \Xi_{T} \subset$ $\mathbb{R}^{L}, L=L_{1}+\ldots+L_{T}$, respectively. Both $\Theta$ and $\Xi$ are assumed to be compact. $P$ is the (regular) joint probability measure of $(\eta, \xi)$ defined on a common Borel space $\left(\Theta \times \Xi, \mathcal{B}^{K+L}\right)$ which must be independent of earlier decisions.

At time $t=0$, a decision $x_{0} \in \mathbb{R}^{n_{0}}$ has to be made without knowing $\left(\eta_{t}, \xi_{t}\right)$ for the subsequent stages $t=1, \ldots, T$. This induces some deterministic costs $\rho_{0}\left(x_{0}\right)$ subject to constraints $f_{0}\left(x_{0}\right) \leq h_{0}$. After an outcome of random data was observed at time $t>0$, the initial policy may be corrected by a new decision $x_{t} \in \mathbb{R}^{n_{t}}$ at stochastic costs $\rho_{t}\left(x^{t}, \eta^{t}\right)$ subject to $f_{t}\left(x^{t}\right) \leq h_{t}\left(\xi^{t}\right)$, based on the history of observations $\eta^{t}:=\left(\eta_{1}, \ldots, \eta_{t}\right), \xi^{t}:=\left(\xi_{1}, \ldots, \xi_{t}\right)$ and previous decisions $x^{t}:=\left(x_{0}, \ldots, x_{t}\right)$. Again, it is emphasized that $x_{t}$ is independent of future outcomes.

A sequence of such nonanticipative decisions $x(\eta, \xi)=\left(x_{0}, x_{1}\left(\eta^{1}, \xi^{1}\right), \ldots\right.$, $\left.x_{T}\left(\eta^{T}, \xi^{T}\right)\right) \in \mathbb{R}^{n}, n=n_{0}+n_{1}+\ldots+n_{T}$, is obtained as the solution of a multistage stochastic program of the form

$$
\begin{array}{ll}
\min & \left\{\rho_{0}\left(x_{0}\right)+\int_{\Theta \times \Xi}\left[\sum_{t=1}^{T} \rho_{t}\left(x^{t}, \eta^{t}\right)\right] d P(\eta, \xi)\right\} \\
\text { s.t. } & f_{0}\left(x_{0}\right) \leq h_{0} \\
& f_{t}\left(x^{t}\right) \leq h_{t}\left(\xi^{t}\right), \quad t=1, \ldots, T, \\
& x(\cdot) \text { nonanticipative. }
\end{array}
$$

Herein, the feasible set is assumed to be compact and non-empty for any outcome of $\xi$. Furthermore, the cost functions $\rho_{t}\left(x^{t}, \eta^{t}\right)$ in $t>0$ are assumed to be convex in $x^{t}$ for any $\eta^{t}$. It can easily be seen that the stochastic program for the management of non-maturing accounts defined in (1), when rewritten as a minimization problem, is exactly of this type. 


\subsection{Saddle property of value functions}

While the functions $\rho_{0}\left(x_{0}\right)$ and $\rho_{t}\left(x^{t}, \eta^{t}\right)$ quantify only those costs that are immediately induced by the sequences of decisions $x^{t}$ and observations $\eta^{t}$, a new decision $x_{t}$ has also an impact on the expected future costs that arise for its correction in the remaining periods $t+1, \ldots, T$. The overall costs that result from $x_{t}$ are given by value functions (with $\phi_{T+1}(\cdot):=0$ )

$$
\begin{aligned}
& \phi_{t}\left(x^{t-1}, \eta^{t}, \xi^{t}\right)=\min _{x_{t}}\left\{\rho_{t}\left(x^{t}, \eta^{t}\right)+\right. \\
& \left.\int_{\Theta_{t+1} \times \Xi_{t+1}} \phi_{t+1}\left(x^{t}, \eta^{t+1}, \xi^{t+1}\right) d P_{t+1}\left(\eta_{t+1}, \xi_{t+1} \mid \eta^{t}, \xi^{t}\right) \mid f_{t}\left(x^{t}\right) \leq h_{t}\left(\xi^{t}\right)\right\}
\end{aligned}
$$

for $t=1, \ldots, T$, where $P_{t}\left(\cdot \mid \eta^{t-1}, \xi^{t-1}\right)$ denotes the conditional probability measure with respect to $\left(\eta_{t}, \xi_{t}\right)$, and the problem for the first stage is

$$
\min _{x_{0}}\left\{\rho_{0}\left(x_{0}\right)+\int_{\Theta_{1} \times \Xi_{1}} \phi_{1}\left(x_{0}, \eta_{1}, \xi_{1}\right) d P\left(\eta_{1}, \xi_{1}\right) \mid f_{0}\left(x_{0}\right) \leq h_{0}\right\}
$$

To ensure that the problems (5) and (6) can be solved, the following conditions for $t=1, \ldots, T$ are sufficient (see [9] for details):

(i) $\Theta_{t} \times \Xi_{t}$ are compact, convex and cover the support of $\left(\eta_{t}, \xi_{t}\right)$,

(ii) $\rho_{t}(\cdot, \cdot)$ are continuous saddle functions, convex in $x^{t}$ and concave in $\eta^{t}$, (iii) $f_{0}(\cdot)$ and $f_{t}(\cdot)$ are convex in $x_{0}$ and $x^{t}, h_{t}\left(\xi^{t}\right)$ is linear-affine in $\xi^{t}$.

Barycentric approximation was originally introduced in [8] in the context of two-stage stochastic programming as a generalization of the inequalities due to Jensen [17] and Edmundson-Madansky [7, 19] that allow for bounding the expectation of saddle functions. According to (5) and (6), the multistage problem (4) can be represented as a series of nested two-stage problems. This motivates the extension to the multistage case.

[Figure 2 about here.] 
Condition $(i)$ implies that problem (5) for the final stage $T$ is a convex optimization problem with parameters $\left(x^{T-1}, \eta^{T}, \xi^{T}\right)$. Since $\phi_{T+1}(\cdot)=0$, assumption (ii) states that the value function $\phi_{T}\left(x^{T-1}, \eta^{T}, \xi^{T}\right)$ is a saddle function which is convex in $\left(x^{T-1}, \xi^{T}\right)$ and concave in $\eta^{T}$. In order to apply the concept to the multistage case, it is necessary that this convex-concave shape of the value function in $T$ is "inherited" to the previous stages $t=$ $T-1, \ldots, 1$. Obviously, preservation of the saddle property is directly related to the type of dependence of $P_{t}$ on $\left(\eta^{t-1}, \xi^{t-1}\right)$ and is not ensured in general. However, one may identify classes of distributions where this is given.

For example, if $P_{t}\left(\eta_{t}, \xi_{t} \mid \eta^{t-1}, \xi^{t-1}\right)=Q_{t}\left(\left(\eta_{t}, \xi_{t}\right)+H_{t}\left(\eta^{t-1}, \xi^{t-1}\right)\right)$, where $Q_{t}$ is a regular distribution function over $\left(\Theta_{t} \times \Xi_{t}, \mathcal{B}^{K_{t}+L_{t}}\right)$ and $H_{t}$ is a linear mapping, the relevant expectation functional takes on the form:

$$
\left(E_{t} \phi_{t}\right)\left(x^{t-1}, \eta^{t}, \xi^{t}\right)=\int \phi_{t}\left(x^{t-1}, \eta^{t-1}, \xi^{t-1},\left(\eta_{t}, \xi_{t}\right)-H_{t}\left(\eta^{t-1}, \xi^{t-1}\right)\right) d Q\left(\eta_{t}, \xi_{t}\right)
$$

Then, it can easily be verified that $E_{t} \phi_{t}$ is a continuous saddle function with respect to its domain (this is the adaption of an argument by Varaiya and Wets [25] for the case where distributions depend on decisions). This results in the additional requirement:

(iv) The distribution function of $P_{t}\left(\cdot \mid \eta^{t-1}, \xi^{t-1}\right)$ depends linearly on the history of observations $\eta^{t-1}$ and $\xi^{t-1}$.

Under this condition, the value function $\phi_{t}\left(x^{t-1}, \eta^{t}, \xi^{t}\right)$ is concave in $\eta^{t}$ and convex in $\xi^{t}$ (see Figure 2 for an illustration of the case that both risk factors are one-dimensional). As discussed in the sequel, this ensures that barycentric approximation provides exact upper and lower bounds also for multistage problems. A more thorough treatment of structural properties and additional conditions for the solvability of the multistage stochastic program can be found in [9]. 
At this point, it should be emphasized that the requirement of a linear dependency has a strong impact on the choice of stochastic processes which are used to describe the dynamics of random data. Clearly, condition (iv) is fulfilled for Brownian motions as introduced in Section 3 since these are independent of the past. However, the saddle property may not be inherited in general for some types of conditional distributions that are postulated by popular term structure models to incorporate a mean reversion in interest rates (see [14] for a discussion on this).

\subsection{Barycentric scenario trees}

In order to exploit the inherent saddle structure of value functions, it is assumed from now on that $\Theta_{t} \subset \mathbb{R}^{K_{t}}$ and $\Xi_{t} \subset \mathbb{R}^{L_{t}}$ are regular simplices. Then, the original probability measure $P_{t}\left(\cdot \mid \eta^{t-1}, \xi^{t-1}\right)$ can be discretized using generalized barycenters that are calculated with respect to the Cartesian product $\Theta_{t} \times \Xi_{t}$ (which is a so-called cross- or $\times$-simplex). Note that the simplices may depend on prior observations $\left(\eta^{t-1}, \xi^{t-1}\right)$ although this is not stressed in the notation for simplicity. If $P_{t}\left(\cdot \mid \eta^{t-1}, \xi^{t-1}\right)$ has unbounded support but some sufficiently small $\epsilon>0$ exists with $P_{t}\left(\Theta_{t} \times \Xi_{t} \mid \eta^{t-1}, \xi^{t-1}\right) \geq 1-\epsilon$, then $P_{t}$ is replaced by its normalized truncation.

Let the vertices of $\Theta_{t}$ and $\Xi_{t}$ be denoted by $u_{\nu_{t}}, \nu_{t}=0, \ldots, K_{t}$, and $v_{\mu_{t}}$, $\mu_{t}=0, \ldots, L_{t}$. Each $\eta_{t} \in \Theta_{t}$ may be represented as a unique convex combination of the points $u_{0}, \ldots, u_{K_{t}}$. The corresponding nonnegative weights $\lambda_{t}\left(\eta_{t}\right):=\left(\lambda_{t, 0}\left(\eta_{t}\right), \ldots, \lambda_{t, K_{t}}\left(\eta_{t}\right)\right)$ are known as barycentric coordinates and given by

$$
\lambda_{t}\left(\eta_{t}\right)=U_{t}^{-1} \cdot\left(\begin{array}{c}
1 \\
\eta_{t}
\end{array}\right) \quad \text { with } U_{t}=\left(\begin{array}{cccc}
1 & 1 & \cdots & 1 \\
u_{0} & u_{1} & \cdots & u_{K_{t}}
\end{array}\right) .
$$

The barycentric coordinates $\tau_{t}\left(\xi_{t}\right):=\left(\tau_{t, 0}\left(\xi_{t}\right), \ldots, \tau_{t, L_{t}}\left(\xi_{t}\right)\right)$ with respect to 
$\Xi_{t}$ are defined analogously:

$$
\tau_{t}\left(\xi_{t}\right)=V_{t}^{-1} \cdot\left(\begin{array}{l}
1 \\
\xi_{t}
\end{array}\right) \quad \text { with } V_{t}=\left(\begin{array}{cccc}
1 & 1 & \cdots & 1 \\
v_{0} & v_{1} & \cdots & v_{L_{t}}
\end{array}\right) .
$$

For each of the $L_{t}$-dimensional simplices $\left\{u_{\nu_{t}}\right\} \times \Xi_{t}$, the probability measure $P_{t}$ induces mass distributions $\mathcal{M}_{\nu_{t}}$ with associated generalized barycenters

$$
\xi_{\nu_{t}}=\left[\mathcal{M}_{\nu_{t}}\left(\left\{u_{\nu_{t}}\right\} \times \Xi_{t}\right)\right]^{-1} \cdot \sum_{\mu_{t}=0}^{L_{t}} v_{\mu_{t}} \int \lambda_{\nu_{t}}\left(\eta_{t}\right) \cdot \tau_{\mu_{t}}\left(\xi_{t}\right) d P_{t}\left(\eta_{t}, \xi_{t} \mid \eta^{t-1}, \xi^{t-1}\right) .
$$

Herein,

$$
\mathcal{M}_{\nu_{t}}\left(\left\{u_{\nu_{t}}\right\} \times \Xi_{t}\right)=\int \tau_{\mu_{t}}\left(\xi_{t}\right) d P_{t}\left(\eta_{t}, \xi_{t} \mid \eta^{t-1}, \xi^{t-1}\right)
$$

is the mass assigned to point $\left(u_{\nu_{t}}, \xi_{\nu_{t}}\right)$. As for $\nu_{t}=0, \ldots, K_{t}$, these mass distributions add up to a probability distribution, one obtains a discrete probability measure $Q_{t}^{l}$ on $\Theta_{t} \times \Xi_{t}$ with support

$$
\operatorname{supp} Q_{t}^{l}=\left\{\left(u_{\nu_{t}}, \xi_{\nu_{t}}\right) \mid \nu_{t}=0, \ldots, K_{t}\right\}
$$

and corresponding probabilities $q_{t}^{l}\left(u_{\nu_{t}}, \xi_{\nu_{t}}\right):=\mathcal{M}_{\nu_{t}}\left(\left\{u_{\nu_{t}}\right\} \times \Xi_{t}\right)$. Due to symmetry, $P_{t}$ also induces mass distributions $\mathcal{M}_{\mu_{t}}$ with associated generalized barycenters

$$
\eta_{\mu_{t}}=\left[\mathcal{M}_{\mu_{t}}\left(\Theta_{t} \times\left\{v_{\mu_{t}}\right\}\right)\right]^{-1} \cdot \sum_{\nu_{t}=0}^{K_{t}} u_{\nu_{t}} \int \lambda_{\nu_{t}}\left(\eta_{t}\right) \cdot \tau_{\mu_{t}}\left(\xi_{t}\right) d P_{t}\left(\eta_{t}, \xi_{t} \mid \eta^{t-1}, \xi^{t-1}\right)
$$

on the $K_{t}$-dimensional simplices $\left(\Theta_{t} \times\left\{v_{\mu_{t}}\right\}\right)$. Again, for $\mu_{t}=0, \ldots, L_{t}$ the mass

$$
\mathcal{M}_{\mu_{t}}\left(\Theta_{t} \times\left\{v_{\mu_{t}}\right\}\right)=\int \lambda_{\nu_{t}}\left(\eta_{t}\right) d P_{t}\left(\eta_{t}, \xi_{t} \mid \eta^{t-1}, \xi^{t-1}\right)
$$

is assigned to point $\left(\eta_{\mu_{t}}, v_{\mu_{t}}\right)$, and the mass distributions $\mathcal{M}_{\mu_{t}}$ add up to a probability distribution. This results in the discrete probability measure $Q_{t}^{u}$ on $\Theta_{t} \times \Xi_{t}$ with support

$$
\operatorname{supp} Q_{t}^{u}=\left\{\left(\eta_{\mu_{t}}, v_{\mu_{t}}\right) \mid \mu_{t}=0, \ldots, L_{t}\right\}
$$


and corresponding probabilities $q_{t}^{u}\left(\eta_{\mu_{t}}, v_{\mu_{t}}\right):=\mathcal{M}_{\mu_{t}}\left(\Theta_{t} \times\left\{v_{\mu_{t}}\right\}\right)$. An illustration of the discretization is shown in Figure 3. The samples represent the joint distribution of $\eta$ and $\xi$ for $K=L=1$ (the time index is omitted for simplicity) which are covered by a $\times$-simplex of rectangular shape in the one-dimensional case. The barycenters $\eta_{0}$ and $\eta_{1}$ can be obtained from projecting the distribution mass onto the simplex that covers the support of $\eta$, taking into account the distance from each sample point to the edges. For each simplex, they are determined as the "center of gravity" of the projected mass, and the probabilities are equivalent to the proportion of the latter to a total mass of one. Performing this procedure for $K=3$ key rates aggregates a great variety of yield curves with different steepness and curvature in only a few points. Likewise, the barycenters $\xi_{0}$ and $\xi_{1}$ result from the projection onto the interval over the domain of the right-hand-side coefficient $\xi$.

[Figure 3 about here.]

The advantageous feature from a computational point of view is that the generalized barycenters in (9), (11) and their probabilities (10), (12) are completely determined by the first moments of $\eta_{t}$ and $\xi_{t}$ as well as the bilinear cross moments $\mathrm{E}\left(\eta_{\nu_{t}} \cdot \xi_{\mu_{t}}\right), \nu_{t}=0, \ldots, K_{t}, \mu_{t}=0, \ldots, L_{t}$. Therefore, the measures $Q_{t}^{l}$ and $Q_{t}^{u}$ incorporate implicitly a correlation between $\eta_{t}$ and $\xi_{t}$ as indicated by the different coordinates of the barycenters in Figure 3. Note that cross moments between different elements of $\eta_{t}$ are not taken into account (the same holds for the components of $\xi_{t}$ ). Hence, equations (9)-(12) do not require any assumption of independence between the random variables at time $t$. Using this approximation scheme dynamically for the conditional 
distributions at all stages, one finally gets two barycentric scenario trees with

$$
\begin{aligned}
& \mathcal{A}_{t}^{l}\left(\eta^{t-1}, \xi^{t-1}\right)=\operatorname{supp} Q_{t}^{l}\left(\cdot \mid \eta^{t-1}, \xi^{t-1}\right) \\
& \mathcal{A}_{t}^{u}\left(\eta^{t-1}, \xi^{t-1}\right)=\operatorname{supp} Q_{t}^{u}\left(\cdot \mid \eta^{t-1}, \xi^{t-1}\right)
\end{aligned}
$$

according to the notation in (2). The corresponding path probabilities are

obtained from (3) when the conditional probabilities are replaced by $q_{t}^{l}$ and $q_{t}^{u}$.

[Figure 4 about here.]

\subsection{Bounds for value functions}

The new measures $Q^{l}$ and $Q^{u}$ provide lower and upper bounds to the multistage stochastic program (4) with the original measure $P$ which can be seen as follows: Substituting the original probability measure $P_{t}$ at each stage by its discrete approximations $Q_{t}^{l}$ and $Q_{t}^{u}$ in (5) yields the value functions

$$
\begin{aligned}
& \psi_{t}\left(x^{t-1}, \eta^{t}, \xi^{t}\right)=\min _{x_{t}}\left\{\rho_{t}\left(x^{t}, \eta^{t}\right)+\right. \\
& \left.\int_{\Theta_{t+1} \times \Xi_{t+1}} \psi_{t+1}\left(x^{t}, \eta^{t+1}, \xi^{t+1}\right) d Q_{t+1}^{l}\left(\eta_{t+1}, \xi_{t+1} \mid \eta^{t}, \xi^{t}\right) \mid f_{t}\left(x^{t}\right) \leq h_{t}\left(\xi^{t}\right)\right\}
\end{aligned}
$$

and

$$
\begin{aligned}
& \Psi_{t}\left(x^{t-1}, \eta^{t}, \xi^{t}\right)=\min _{x_{t}}\left\{\rho_{t}\left(x^{t}, \eta^{t}\right)+\right. \\
& \left.\int_{\Theta_{t+1} \times \Xi_{t+1}} \Psi_{t+1}\left(x^{t}, \eta^{t+1}, \xi^{t+1}\right) d Q_{t+1}^{u}\left(\eta_{t+1}, \xi_{t+1} \mid \eta^{t}, \xi^{t}\right) \mid f_{t}\left(x^{t}\right) \leq h_{t}\left(\xi^{t}\right)\right\}
\end{aligned}
$$

with $\psi_{T+1}(\cdot)=\Psi_{T+1}(\cdot):=0$ for $t=1, \ldots, T$. Both $\psi_{t}$ and $\Psi_{t}$ are bilinear functions since the integrand $\lambda_{\nu_{t}}\left(\eta_{t}\right) \cdot \tau_{\mu_{t}}\left(\xi_{t}\right)$ in (9) and (11) is bilinear in $\left(\eta_{t}, \xi_{t}\right)$. It is shown in [9] that the following relation holds:

$$
\psi_{t}\left(x^{t-1}, \eta^{t}, \xi^{t}\right) \leq \phi_{t}\left(x^{t-1}, \eta^{t}, \xi^{t}\right) \leq \Psi_{t}\left(x^{t-1}, \eta^{t}, \xi^{t}\right)
$$

The meaning of this inequality is that the value function $\phi_{t}(\cdot)$ with respect to the original measure $P_{t}$ (which is a saddle function) is supported from 
below and above by two bilinear functions $\psi_{t}(\cdot)$ and $\Psi_{t}(\cdot)$ with respect to the approximated measures $Q_{t}^{l}$ and $Q_{t}^{u}$. In particular, the supporting points for the minorant are the barycenters $\xi_{\nu_{t}}, \nu_{t}=0, \ldots, K_{t}$, while the majorant supports the value function in $\eta_{\mu_{t}}, \mu_{t}=0, \ldots, L_{t}$ (see Figure 4 for the case $K=L=1$ where again the time index is omitted for simplicity). Obviously, the two bilinear functions $\psi_{t}$ and $\Psi_{t}$ can now easily be integrated since the calculation of the expectations reduces to the weighted sums

$$
\begin{aligned}
& \mathrm{E}_{t} \psi_{t}\left(x^{t-1}, \eta^{t}, \xi^{t}\right)=\sum_{\left(\eta_{t}, \xi_{t}\right) \in \mathcal{A}_{t}^{l}} \psi_{t}\left(x^{t-1}, \eta^{t}, \xi^{t}\right) \cdot q_{t}^{l} \\
& \mathrm{E}_{t} \Psi_{t}\left(x^{t-1}, \eta^{t}, \xi^{t}\right)=\sum_{\left(\eta_{t}, \xi_{t}\right) \in \mathcal{A}_{t}^{u}} \Psi_{t}\left(x^{t-1}, \eta^{t}, \xi^{t}\right) \cdot q_{t}^{u}
\end{aligned}
$$

which was the intention of the approximation. Again, note that according to (13) the barycentric scenario trees $\mathcal{A}^{l}$ and $\mathcal{A}^{u}$ are constructed by combination of the vertices of the $\Theta_{t}$-simplex and the barycenters with respect to $\Xi_{t}$ for the lower bound and vice versa in case of the upper bound. Due to the incorporation of vertices of the support of random data, it is ensured that "extremal events" are taken into account in the scenarios. From a practical point of view, this may be seen as particularly useful to hedge against the various risks. For example, liquidity can always be preserved under the dynamic investment strategies since the constraints in (1) must also hold for the scenario with the largest drop in volume, provided that the underlying stochastic processes and their parameter estimates describe the evolution of risk factors properly.

The two approximate problems that result from the substitution of the original measure $P$ in (4) by $Q^{l}$ and $Q^{u}$ can be treated as deterministic multistage programs. Their solution provides policies $x^{l}:=\left(x_{0}^{l}, \ldots, x_{T}^{l}\right)$ and $x^{u}:=\left(x_{0}^{u}, \ldots, x_{T}^{u}\right)$, where $x_{t}^{l}$ and $x_{t}^{u}$ denote the decisions made after $\left(\eta_{t}^{l}, \xi_{t}^{l}\right) \in$ $\mathcal{A}_{t}^{l}$ and $\left(\eta_{t}^{u}, \xi_{t}^{u}\right) \in \mathcal{A}_{t}^{u}$ are observed in $t=1, \ldots, T$. Since the decisions in 
$t>0$ correspond to outcomes in the barycentric scenarios that may be seen as "representative" rebalancing actions and are not likely to be implemented, only the first-stage policy in $t=0$ is of interest for the user. However, a situation may occur where the solutions of the lower and upper approximated problems are not unique, i.e., $x_{0}^{l} \neq x_{0}^{u}$.

In this case, a refinement of the approximation and, hence, more accurate bounds can be achieved by partitioning the $\times$-simplices into subsimplices. Under weak convergence of the discrete measures $Q_{t}^{l}, Q_{t}^{u}$ to $P_{t}$, epi-convergence of the approximated value functions $\psi_{t}$ and $\Psi_{t}$ to $\phi_{t}$ in $t=1, \ldots, T$ is guaranteed when the sub-simplices become arbitrarily small with respect to diameter (see [9] for details). However, this refinement process must be controlled carefully because each partition increases the size of the corresponding scenario tree.

To this end, the accuracy of the discretization is quantified by the difference $\epsilon_{t}\left(\eta^{t}, \xi^{t}\right):=\Psi_{t}\left(x^{t-1}, \eta^{t}, \xi^{t}\right)-\psi_{t}\left(x^{t-1}, \eta^{t}, \xi^{t}\right)$ between the upper and lower bound. Then, a partition can be performed in the node with the largest approximation error $\epsilon_{t}(\cdot)$. The corresponding approximated problems are solved until the desired accuracy is achieved which results in a sequence of successively increasing deterministic equivalent programs. If $\epsilon_{t}(\cdot)=0$ for some node, the approximation of $\phi_{t}$ is exact there, and a (further) partitioning of the associated $\times$-simplex does not improve the accuracy of the solution. In this sense, the existence and utilization of exact bounds may be seen as one of the most important features of barycentric approximation for the solution of multistage stochastic programs (see [12] for a discussion of refinement techniques).

[Figure 5 about here.] 


\section{$5 \quad$ Performance of investment policies}

The model for savings deposits (1) as well as its equivalent for refinancing variable-rate mortgages was developed and implemented in cooperation with a major Swiss bank. Before the bank decided to use the models for the management of their non-maturing account positions, case studies were conducted to assess their performance and compare it with the replicating portfolio approach that was employed up to this time. In case of savings accounts, the study was based on monthly interest data (money market and swap rates) for the Swiss Franc from March 1989 to April 1996. The corresponding volume was taken from the aggregated savings positions of all financial institutions in Switzerland and the Principality of Liechtenstein as stated in the monthly reports of the Swiss National Bank and scaled to an initial volume of 30 billion (see Figure 5).

Investment opportunities consisted of money market and swap positions with maturities of $1,2,3,4,5,7$, and 10 years. Short sales were not permitted, upper bounds for new investments were set to 500 million for maturities up to 5 years and 200 million for 7 and 10 years in order to take liquidity restrictions on the Swiss interbank market into account. For the initial portfolio composition, it was assumed that a replicating portfolio rule had been applied before with $50 \%$ two-year and $50 \%$ five-year instruments (see Section 2.1), i.e., an amount of 875 million matures in each of the first 24 months and 250 million in each of the following 36 months.

Practical experience showed that it is helpful to base an investment decision not only on a single optimization run where scenarios are determined with expected values of zero for the underlying multi-dimensional stochastic processes. Instead, a set of "stress scenarios" characterized by various key rate drifts was defined that reflect shift and tilt movements of the yield 
curve. In general, the optimization runs for the entire set of key rate drifts result in 3-5 different optimal investment policies and corresponding present values of the income from interest payments. This allows an assessment of the sensitivity of the solution with respect to different assumptions for the trend of interest rates and, in particular, to quantify the impact of "extremal events" defined by the stress scenarios whose magnitude is not captured by the stochastic processes on which the discretization was based.

To this end, the different first-stage decisions obtained from all optimization runs are fixed, and the optimization is repeated with respect to the remaining stages for all combinations of key rate drifts. A comparison with the objective function values of the unrestricted problems quantifies the consequences of a suboptimal initial decision and the costs for their correction in the subsequent periods. This reveals the risk associated with the different solutions with respect to non-anticipated shift and tilt movements of the term structure. In this way, a profit and loss pattern is derived that helps identify dominant policies for the first-stage decision (see [13] for more details).

[Figure 6 about here.]

This procedure is performed for all months out of the sample period which results in some kind of roll-over planning. The evolution of the margin between the return of the portfolio and the client rate over the entire sample period is shown in Figure 6 for the dynamic policies obtained from the multistage stochastic programming model, followed by the risk analysis described above, and compared with two replicating portfolio strategies. While the weights of RP1 were derived using the real fluctuations of the savings position under management, the composition of RP2 was determined under the assumption of a constant volume, i.e., it tracks only the client rate and 
ignores the volume risk. Note that this results in a lower and more volatile margin.

[Table 1 about here.]

According to Table 1, the average margin from 1989-1996 could be improved by 25 basis points compared to the better static policy RP1. In particular, the margin's standard deviation was reduced significantly although it is not considered explicitly in the objective of the stochastic program in contrast to the determination of replicating portfolios by minimization of the tracking error. However, volatility of the risk factors is incorporated by the large number of scenarios in the tree of the stochastic program. Similar observations have been made for other stochastic optimization models (see [3] for example).

\section{Conclusions and outlook}

In this paper, a multistage stochastic programming model was introduced for the management of non-maturing account positions like savings and sight deposits, variable-rate mortgages etc. The solvability of the stochastic program suffers from the multi-dimensional integration and nested optimization of the implicitly given value functions. In order to overcome these numerical difficulties, the latter are approximated from below and above by bilinear functions. Under certain conditions for the type of constraints and underlying (conditional) probability distributions, the solution of the corresponding deterministic equivalent programs provides exact lower and upper bounds to the original problem. Furthermore, the accuracy of the approximation can be quantified and improved by refinement techniques that are currently under investigation. 
The enhanced performance of the model compared to the replicating portfolio approach clearly shows that a dynamic policy is superior to static strategies. Various sources of risk can be taken into account by generation of "representative" scenarios for which the impact of future rebalancing actions is evaluated. Since these scenarios are based on extremal measures by construction, large changes in interest rates and volume are explicitly considered. In particular, this allows to hedge against the liquidity or prepayment risk inherent to non-maturing accounts by dynamic portfolio strategies. Moreover, a dependency between interest rates and volume may be incorporated by the correlations between both. The increase in margin achieved in the case study is confirmed by the experience made at a major Swiss bank where the model has been applied to the management of variable-rate mortgages since 1995 and savings deposits since 1997.

A promising way for an additional improvement of the model and, possibly, its performance can be seen in a more appropriate description of the risk factor dynamics. For example, interest rates tend to fluctuate around a longterm mean over a horizon of several years. This mean reversion property is incorporated by most of the common term structure models that have been proposed in the financial literature. An examination of alternative interest rate models and their use for scenario generation with empirical results for the Swiss Franc can be found in [14]. A trend-stationary process for the evolution of savings deposits is proposed in [23]. In this case, using the factors of the term structure model as explanatory variables provides a good long-term description for the aggregated savings volume published by the Swiss National Bank. When barycentric approximation is applied to the construction of scenario trees, the choice of distributions for the random data is restricted since the saddle property of value functions on which the bounds are based 
is not inherited in general.

In addition to "realistic" distribution assumptions for the risk factors, the determination of the "correct" set of parameters is essential. The analysis in [14] and [23] based on maximum likelihood estimation reveals that all term structure models under investigation provide a bad fit to empirically observed quantiles of interest rates, do not match the curtosis of their changes etc. However, it is crucial for risk management systems that the tails of the distributions are captured accurately. An extension of simulated moment estimation proposed by Mulvey et al. [22] allows for a closer match between some target moments and the moments of simulated processes while the relative importance of certain statistics may be chosen by the decision maker, depending on particular requirements of the application. Unfortunately, there is not much literature that addresses the importance of a proper description of the risk factor dynamics and accurate calibration of model parameters in the context of scenario generation for stochastic optimization models.

The model for non-maturing accounts presented here may be seen as a first step towards a general ALM model for optimal investment and refinancing strategies under consideration of the interest rate and volume risk in a bank's balance. It may also be extended by additional constraints, e.g., limits for the absolute risk exposure of certain positions in order to comply with internal risk models or regulatory restrictions concerning capital requirements. In this sense, multistage stochastic programming is a versatile tool for financial planning problems under uncertainty that helps overcome many deficiencies of static approaches.

Acknowledgement: Research for this paper was supported by the Swiss National Science Foundation, Grant No. 21-39'575.93. 


\section{References}

[1] A. Beltratti, A. Consiglio, S.A. Zenios, Scenario modeling for the management of international bond portfolios, Annals of Operations Research, 85 (1999) 227-247.

[2] J.R. Birge, R.J.-B. Wets, Designing approximation schemes for stochastic optimization problems, in particular for stochastic programs with recourse, Mathematical Programming Study, 27 (1986) 54-102.

[3] D.R. Cariño, W.T. Ziemba, Formulation of the Russell-Yasuda Kasai financial planning model, Operations Research, 46 (1998) 433-449.

[4] G.B. Dantzig, G. Infanger, Multi-stage stochastic linear programs for portfolio optimization, Annals of Operations Research, 45 (1993) 59-76.

[5] N.C.P. Edirisinghe, New second-order bounds on the expectation of saddle functions with applications to stochastic linear programming, Operations Research, 44 (1996) 909-922.

[6] N.C.P. Edirisinghe, W.T. Ziemba, Bounds for two-stage stochastic programs with fixed recourse, Mathematics of Operations Research, 19 (1994) 292-313.

[7] H.P. Edmundson, Bounds on the expectation of a convex function of a random variable, Technical Report 982, RAND Corporation, Santa Monica, 1957.

[8] K. Frauendorfer, Stochastic Two-Stage Programming, Springer, Berlin, 1992. 
[9] K. Frauendorfer, Multistage stochastic programming: Error analysis for the convex case, Mathematical Methods of Operations Research, 39 (1994) 93-122.

[10] K. Frauendorfer, Barycentric scenario trees in convex multistage stochastic programming, Mathematical Programming (Series B), 75 (1996) 277-293.

[11] K. Frauendorfer, P. Kall, A solution method for SLP recourse problems with arbitrary multivariate distributions - the independent case, Problems of Control and Information Theory, 17 (1988) 177-205.

[12] K. Frauendorfer, C. Marohn, Refinement issues in stochastic multistage linear programming, in: K. Marti, P. Kall (Eds.), Stochastic Programming Methods and Technical Applications (Proceedings of the 3rd GAMM/IFIP Workshop 1996), Springer, Berlin, 1998, pp. 305-328.

[13] K. Frauendorfer, M. Schürle, Stochastic optimization in asset \& liability management: A model for non-maturing accounts, in: S.P. Uryasev (Ed.), Probabilistic Constrained Optimization: Methodology and Applications, Kluwer, Norwell, 2000, pp. 67-101.

[14] K. Frauendorfer, M. Schürle, Term structure models in multistage stochastic programming: Estimation and approximation, Annals of $O p$ erations Research, 100 (2000) 189-209.

[15] B. Golub, M. Holmer, R. McKendall, L. Pohlman, S.A. Zenios, A stochastic programming model for money management, European Journal of Operational Research, 85 (1995) 282-296.

[16] T. Ho, Key rate durations: Measures of interest rate risks, Journal of Fixed Income, 2 (1992) 29-44. 
[17] J.L.W.V. Jensen, Sur les fonctions convexes et les inégalités entre les valeurs moyennes, Acta Mathematica, 30 (1906) 175-193.

[18] S.T. Kauth, Risikomanagement von Struktureffekten im Bankgeschäft, Haupt, Bern, 1997.

[19] A. Madansky, Bounds on the expectation of a convex function of a multivariate random variable, Annals of Mathematical Statistics, 30 (1959) 743-746.

[20] J.M. Mulvey, Financial planning via multi-stage stochastic programs, in: J.R. Birge, K.G. Murty (Eds.), Mathematical Programming: State of the Art 1994, University of Michigan, Ann Arbor, 1994, pp. 151-171.

[21] J.M. Mulvey, Generating scenarios for the Towers Perrin investment systems, Interfaces, 26 (1996) 1-15.

[22] J.M. Mulvey, D.P. Rosenbaum, B. Shetty, Parameter estimation in stochastic scenario generation systems, European Journal of Operational Research, 118 (1999) 563-577.

[23] M. Schürle, Zinsmodelle in der stochastischen Optimierung, Haupt, Bern, 1998.

[24] D.G. Uyemura, D.R. van Deventer, Risk Management in Banking, McGraw-Hill, New York, 1993.

[25] P. Varaiya, R.J.-B. Wets, Stochastic dynamic optimization: Approaches and computation, in: M. Iri, K. Tanabe (Eds.), Mathematical Programming: Recent Developments and Applications, Kluwer, Norwell, 1989, pp. 309-332. 


\section{List of Figures}

1 Scenario tree (a) and nonanticipativity constraints (b) . . . . 31

2 Saddle property of the value function . . . . . . . . . . . 32

3 Approximation of a correlated distribution $(K=L=1)$. . . 33

4 Bilinear approximations of the value function . . . . . . . 34

$5 \quad$ Interest rates (left) and savings volume (billion CHF, right) . . 35

6 Margin of two replicating portfolios and optimization model . 36 




(a)

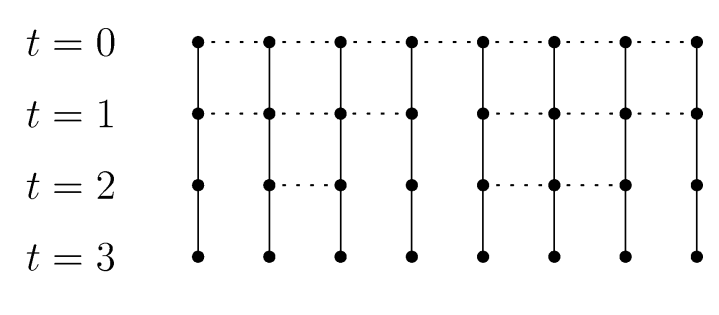

(b)

Figure 1: Scenario tree (a) and nonanticipativity constraints (b) 




Figure 2: Saddle property of the value function 


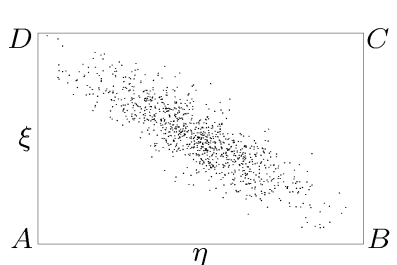

(a) $\times$-simplex

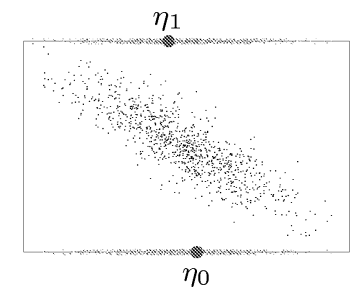

(b) Barycenters for $\eta$

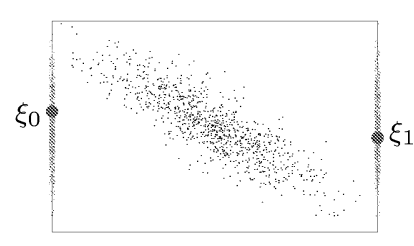

(c) Barycenters for $\xi$

Figure 3: Approximation of a correlated distribution $(K=L=1)$ 


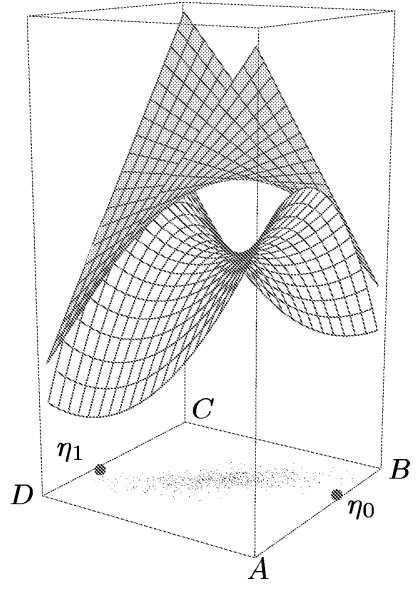

(a) Upper bound

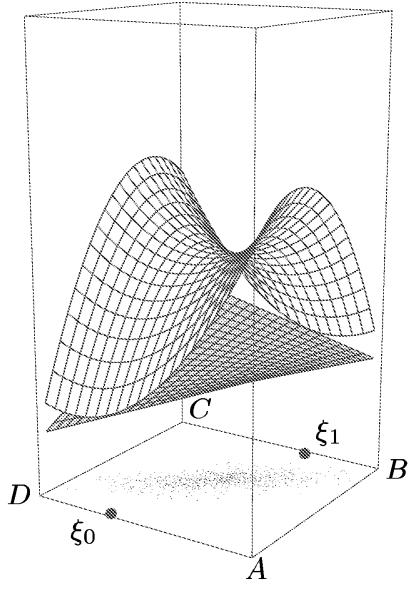

(b) Lower bound

Figure 4: Bilinear approximations of the value function 




Figure 5: Interest rates (left) and savings volume (billion CHF, right) 




Figure 6: Margin of two replicating portfolios and optimization model 


\section{List of Tables}

1 Comparison of dynamic policy with replicating portfolios . . . 38 


\begin{tabular}{ccccccc}
\hline \multirow{2}{*}{ policy } & \multicolumn{3}{c}{ weights } & & \multicolumn{2}{c}{ margin [\%] } \\
\cline { 2 - 4 } \cline { 7 - 7 } & 1 yr & 2 yrs & 5 yrs & & mean & std. dev. \\
\hline SO model & - & - & - & & 2.659 & 0.188 \\
RP 1 & 0.0 & 0.5 & 0.5 & & 2.414 & 0.358 \\
RP 2 & 0.35 & 0.35 & 0.3 & & 2.399 & 0.696 \\
\hline
\end{tabular}

Table 1: Comparison of dynamic policy with replicating portfolios 